\title{
Acute extrapyramidal syndrome in mild ornithine transcarbamylase deficiency: metabolic stroke involving the caudate and putamen without metabolic decompensation
}

Received: 26 July 2002/ Revised: 28 October 2002 / Accepted: 5 November 2002/Published online: 7 February 2003

(C) Springer-Verlag 2003

\begin{abstract}
A 6-year-old male with partial ornithine transcarbamylase (OTC) deficiency had acute and rapidly progressive symmetrical swelling of the head of the caudate nuclei and putamina. Clinical presentation was ataxia and dysarthria progressing to seizures and coma; these symptoms gradually resolved with supportive management. Although he had been recently treated for mild hyperammonemia, there was no evidence of acute metabolic decompensation prior to presentation, and plasma ammonia and amino acids were consistent with good metabolic control. This case is novel in that the neurological insult affected the neostriatum of the basal ganglia and the episode occurred in the absence of an apparent metabolic abnormality, unique observations in a patient with OTC deficiency. Conclusion: This case suggests that the pathophysiology of metabolic stroke is complicated. It also argues for an evaluation for metabolic stroke in patients with known inborn errors of metabolism who present with unusual neurological symptoms in the absence of biochemical abnormalities. Similarly, this case suggests that patients presenting with unexplained neurological insults might benefit from an evaluation for an inborn error of metabolism.
\end{abstract}

C.E. Keegan · D.M. Martin · J.L. Gorski $(\bowtie)$

Department of Pediatrics and Communicable Diseases,

Division of Pediatric Genetics, University of Michigan

School of Medicine, 3570 MSRB II, P.O. Box 0688 ,

Ann Arbor, MI, 48109-0688, USA

E-mail: jlgorski@umich.edu

Tel.: + 1-734-7635276

Fax: +1-734-7639512

D.M. Martin · J.L. Gorski

Department of Human Genetics, University of

Michigan Medical School, 48109 Ann Arbor, MI

D.J. Quint

Department of Radiology, University of Michigan Medical School, 48109 Ann Arbor, MI
Keywords Metabolism - Amino acid metabolism Inborn errors $\cdot$ Basal ganglia $\cdot$ Neostriatum

Abbreviations $O T C$ Ornithine transcarbamylase $\cdot C P S$ Carbamyl phosphate synthetase $\cdot$ MELAS

Mitochondrial myopathy-encephalopathy-lactic acidosis and stroke-like episodes

\section{Introduction}

Ornithine transcarbamylase (OTC) deficiency (MIM 311250 ) is the most common urea cycle disorder, with an estimated incidence of $1 / 14,000$ [2]. Urea cycle disorders usually present in the neonatal period with encephalopathy and hyperammonemia (typically greater than $200 \mu \mathrm{M}$; normal $11-35 \mu \mathrm{M}$ ) without increased anion gap. Older patients with hyperammonemia due to metabolic decompensation commonly present with ataxia, dysarthria and/or mental status changes. Hyperammonemia is a medical emergency; untreated it leads to astrocyte swelling and generalized cerebral edema [2]. Unless treatment is rapidly instituted, developmental delay and mental retardation are likely; neurological outcome is correlated with the frequency and duration of hyperammonemic encephalopathy [17, 18, 23].

Metabolic stroke, defined as acute focal neurological deficits with correlated lesions on brain imaging, is a well-documented but poorly understood complication of certain inborn errors of metabolism. In previously reported patients, these episodes were typically accompanied by acute metabolic decompensation with significant biochemical abnormalities $[4,5,11,12,13,15,20,22]$. Some cases were reversible $[4,12,20,22]$, while other cases were fatal $[5,8,9,10,13]$. Here we describe a patient with OTC deficiency who, despite evidence of good metabolic control at the onset of symptoms, developed a reversible neurological insult to the neostriatum (caudate and putamen). This case is novel; to our knowledge, acute reversible insults to the neostriatum have not been reported in patients with OTC deficiency. 
This observation suggests that factors other than acute systemic metabolic decompensation may account for this stroke-like syndrome.

\section{Case History}

D.A. was born to a 31-year-old woman who had a confirmed family history of OTC deficiency (Fig. 1). Biochemical abnormalities consistent with the diagnosis of OTC deficiency, including hyperammonemia, elevated plasma glutamine and urinary orotic acid with low plasma arginine and citrulline were noted in infancy. Previous mutational analyses of the OTC gene in a maternal cousin showed a $\mathrm{Y} 202 \mathrm{H}$ missense mutation (CAC $>$ TAC) (Fig. 1); this mutation was reported in an unrelated individual with OTC deficiency who presented at 2 years of age with hyperammonemia and encephalopathy [1]. Diagnostic biochemical abnormalities, a family history of OTC deficiency, and DNA diagnostic studies in an affected maternal cousin confirmed the diagnosis of OTC deficiency in our patient.

D.A. had numerous hospitalizations for hyperammonemia presenting as lethargy and transient ataxia; these had been successfully treated with protein restriction and intravenous sodium benzoate and sodium phenylacetate. Laboratory values from a typical hospitalization at age 5.5 years are shown in Table 1. D.A. had delayed developmental milestones; at age 4 , he was ambulatory and had minimal expressive language. By age 5, he used utensils and was toilet trained. He was diagnosed with attention deficit hyperactivity disorder and had significant behavioral problems including property destruction and the mistreatment of animals.

At age 5 and 11/12 years, he presented with a broad based gait, hyperammonemia $(131 \mu \mathrm{M})$, and a presumed viral infection. He was treated with intravenous sodium benzoate, sodium phenylacetate, and arginine and was discharged after a 3-day hospitalization with a normal neurological examination and a normal ammonia level. Twenty-four hours after discharge, he presented with acute onset of ataxia, drooling, and slurred speech without associated symptoms. His ammonia was $22 \mu \mathrm{M}$ (normal, 11-35). Plasma glutamine was moderately elevated at $721 \mu \mathrm{M}$ (normal, 123-600); citrulline and arginine were within normal limits. Essential amino acids, albumin, and total protein were normal, reflecting adequate nutritional status and liver protein synthesis (Table 1). A non-contrast head CT scan was normal, with no evidence of acute hemorrhage, ischemia, or infarction. He remained stable

Fig. 1 Four-generation pedigree showing males $(\square)$, females $(\bigcirc)$, and obligate carrier females $(\odot)$. $P$ indicates the propositus, D.A. (IV-11). Individuals III-8, -10 , and -23 and IV-6, $-8,-10$, and -11 had biochemical evidence of OTC; individual IV-13 had a Y202H missense OTC mutation. Males who died in infancy (individuals II3 , III-3, -4, -15, -16, and IV-5) are presumed to have had OTC deficiency. Spontaneous abortions are indicated $(\perp)$. Ages are given for deceased individuals (III-12) or infants (inf) metabolically, but worsening ataxia and dysarthria prompted a brain MRI on hospital day 2, which showed mild increased signal intensity in the putamina and head of the caudate nuclei, consistent with gray matter swelling (Fig. 2A). On hospital day 3, he developed right-sided seizures and coma. He had brisk deep tendon reflexes, ankle clonus and a left Babinski sign; intermittent choreiform movements of the arms were noted. Repeat non-contrast head CT scans on hospital days 3 and 4 showed subtle interval development of decreased attenuation in the basal ganglia, consistent with focal edema (Fig. 2B). There was no evidence of diffuse cerebral edema, acute hemorrhage or ischemia. A prothrombin time was slightly elevated; serum albumin and total protein remained normal, indicating normal liver function (Table 1).

Just prior to the onset of seizures, D.A.'s ammonia level was $98 \mu \mathrm{M}$ (Table 1). He was treated with intravenous sodium benzoate, sodium phenylacetate and arginine, and his ammonia level quickly normalized. During his acute ataxia and seizures, his plasma glutamine was normal $(267 \mu \mathrm{M})$ and he had a normal urine organic acid profile. Serial arterial lactate and pyruvate measurements during his hospitalization were normal. CSF for lactate and quantitative amino acids were also normal (Table 1). Additionally, mitochondrial DNA mutational analyses for tRNA leucine mutations associated with MELAS (mitochondrial myopathyencephalopathy-lactic acidosis and stroke-like episodes; MIM 540000) were normal. Lumbar puncture showed normal white and red blood cell counts, protein, glucose, Gram stain and culture. Blood and CSF viral cultures, and CSF PCR for herpes simplex virus were negative. A blood lead level, carboxyhemoglobin determination, and hair analysis for heavy metals were normal; an HIV antibody test was also negative.

An EEG on hospital day 4 showed diffuse suppression, consistent with moderate encephalopathy. Repeat brain MRI on hospital day 9 showed increased signal intensity in the head of the caudate nuclei and putamina, consistent with progression of the edema in these regions (Fig. 2C and D). His neurological status gradually improved with supportive care, and he was extubated on hospital day 15. At discharge on hospital day 33, he was eating normally, speaking with some dysarthria, walking with assistance, and riding a tricycle. After 3 additional weeks, he had returned to his baseline behavioral and neurological status; he was walking, running, and speaking in full sentences. He continued to have mild difficulties with balance and a resting hand tremor.

\section{Discussion}

We present a 6-year-old male with partial OTC deficiency who acutely developed edema of the neostriatum with correlative symptoms in the setting of normal biochemical parameters. The presenting symptoms of ataxia and dysarthria were more severe than those previously observed during previous episodes of hyperammonemia. His ammonia at the time of presentation was

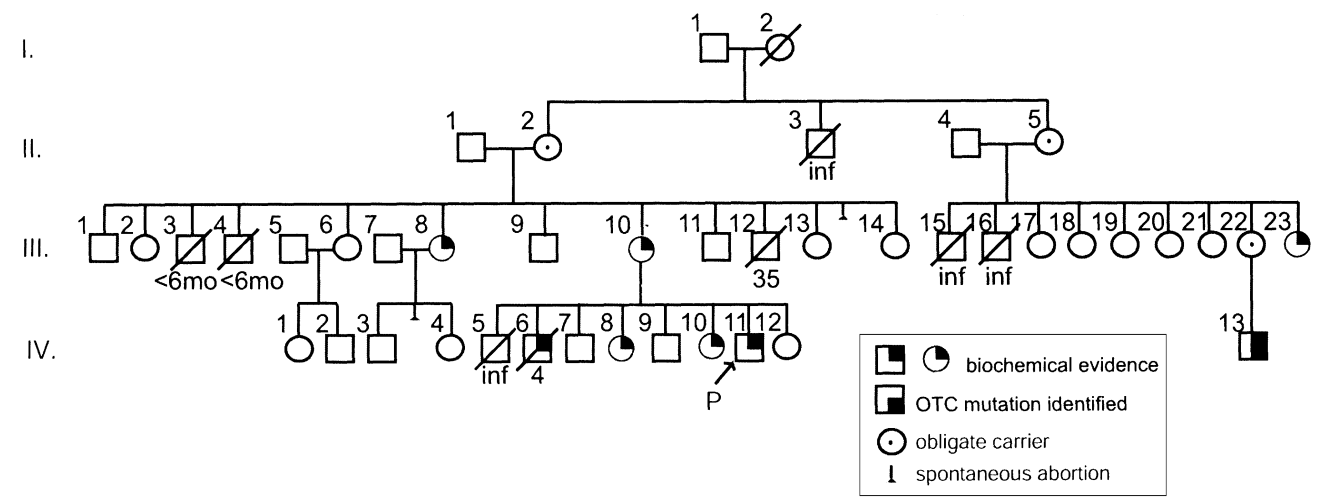


Table 1 Blood and CSF laboratory values

\begin{tabular}{|c|c|c|c|c|c|c|c|}
\hline & $\begin{array}{l}\text { Past hospi- } \\
\text { talization }\end{array}$ & $\begin{array}{l}3 \text { days prior } \\
\text { to episode }\end{array}$ & $\begin{array}{l}\text { Acute } \\
\text { episode }\end{array}$ & $\begin{array}{l}\text { Onset of } \\
\text { seizures }\end{array}$ & $\begin{array}{l}\text { Day of } \\
\text { discharge }\end{array}$ & $\begin{array}{l}3 \text { weeks following } \\
\text { discharge }\end{array}$ & $\begin{array}{l}\text { Normal } \\
\text { values }\end{array}$ \\
\hline $\mathrm{NH}^{\mathrm{a}}$ & 264 & 131 & 22 & 98 & 12 & 17 & $11-35$ \\
\hline Glutamine $^{\mathrm{a}}$ & 1207 & $910^{\mathrm{d}}$ & 721 & 267 & 471 & 499 & $123-600$ \\
\hline Alanine $^{\mathrm{a}}$ & 185 & $1210^{\mathrm{d}}$ & 843 & 510 & 682 & 303 & $141-343$ \\
\hline Citrulline $^{\mathrm{a}}$ & 1 & $76^{\mathrm{d}}$ & 26 & 6 & 22 & 13 & $2-41$ \\
\hline Arginine $^{\mathrm{a}}$ & 42 & $74^{\mathrm{d}}$ & 78 & 112 & 136 & 47 & $40-145$ \\
\hline Threonine $^{\mathrm{a}}$ & 45 & $110^{\mathrm{d}}$ & 95 & 42 & 66 & 157 & $85-196$ \\
\hline Valine $^{\mathrm{a}}$ & 99 & $116^{\mathrm{d}}$ & 177 & 55 & 47 & 130 & $112-284$ \\
\hline Cystine $^{\mathrm{a}}$ & 33 & $25^{\mathrm{d}}$ & 30 & 39 & 40 & 18 & $3-48$ \\
\hline Isoleucine $^{a}$ & 34 & $25^{\mathrm{d}}$ & 37 & 12 & 10 & 43 & $35-79$ \\
\hline Leucine $^{\mathrm{a}}$ & 59 & $40^{\mathrm{d}}$ & 79 & 22 & 18 & 72 & $60-168$ \\
\hline Phenylalanine $^{\mathrm{a}}$ & 33 & $48^{\mathrm{d}}$ & 42 & 37 & 24 & 31 & $30-70$ \\
\hline Tryptophan $^{\mathrm{a}}$ & 24 & $0^{\mathrm{d}}$ & 18 & 17 & 22 & 20 & $0-123$ \\
\hline Lysine $^{\mathrm{a}}$ & 207 & $164^{\mathrm{d}}$ & 141 & 75 & 92 & 102 & $81-213$ \\
\hline Histidine $^{\mathrm{a}}$ & 77 & $94^{\mathrm{d}}$ & 61 & 65 & 45 & 54 & $47-104$ \\
\hline Lactate $^{\mathrm{b}}$ & - & - & - & 0.6 & - & - & $0.5-2.2$ \\
\hline Pyruvate ${ }^{\mathrm{b}}$ & - & - & - & 0.05 & - & - & $0.03-0.15$ \\
\hline CSF glutamine ${ }^{a}$ & - & - & - & 454 & - & - & $161-533$ \\
\hline CSF lactate ${ }^{b}$ & - & - & - & 2.2 & - & - & $0.5-2.2$ \\
\hline Albumin ${ }^{c}$ & 4.8 & 4.5 & 3.9 & 3.4 & 3.2 & - & $3.2-5.2$ \\
\hline Total protein ${ }^{\mathrm{c}}$ & 7.7 & 7.2 & 6.5 & 6.1 & - & - & $5.8-8$ \\
\hline Prothrombin ${ }^{\mathrm{e}}$ time/INR & - & - & - & $11.8 / 1.2$ & - & - & $9.3-11.1$ \\
\hline
\end{tabular}

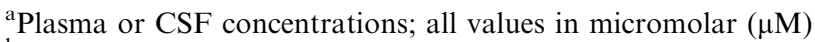

${ }^{\mathrm{b}}$ Serum or CSF concentrations in milliequivalents/liter

${ }^{\mathrm{c} S}$ Serum concentrations in grams/deciliter

${ }^{\mathrm{d}}$ Values obtained 2 weeks prior to presentation

${ }^{\mathrm{e}}$ Values are in seconds. Dashes (-) denote values not obtainedf

in the normal range and his glutamine was only modestly elevated. Essential amino acids, total protein and serum albumin concentrations were normal, reflecting adequate nutritional status. Although he had recently
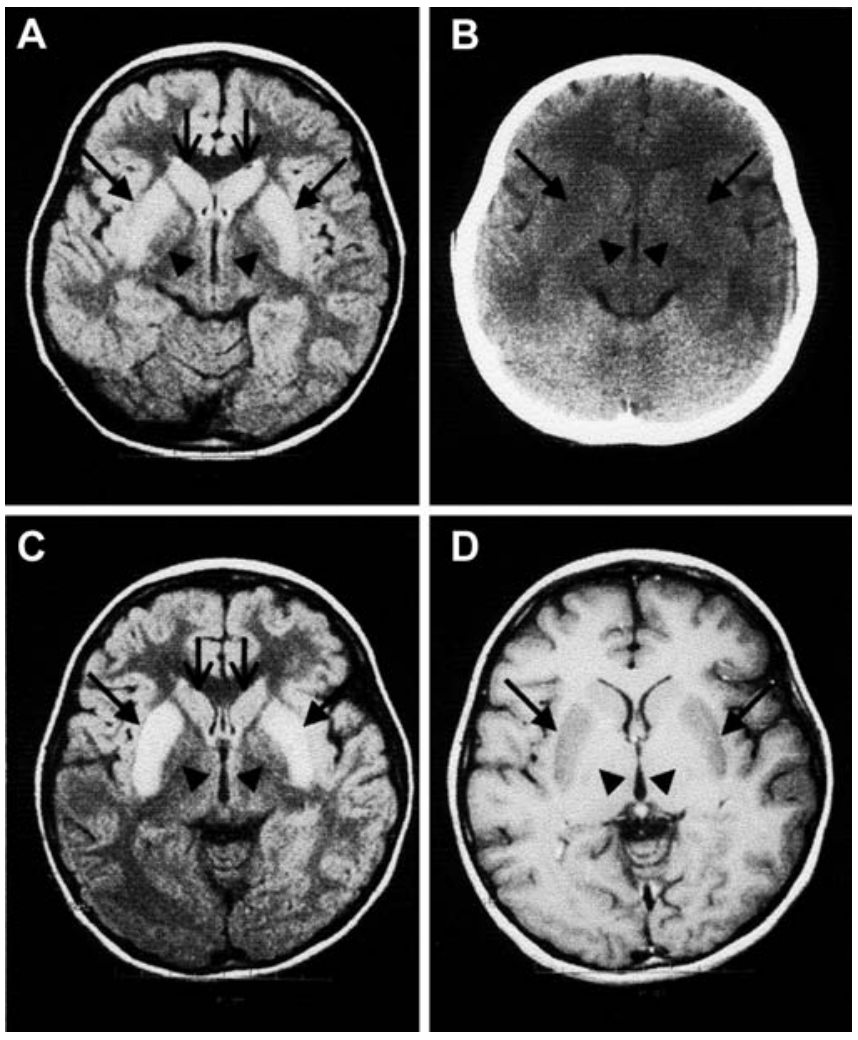

been hospitalized with mild hyperammonia, he had returned to his baseline status $24 \mathrm{~h}$ prior to the onset of his new acute symptoms. Head MRI scans showed evidence of injury to the basal ganglia, specifically in the head of the caudate nuclei and the putamina. Ultimately, his neurological status improved and he was left with minimal residual neurological deficits. To our knowledge, metabolic stroke affecting the basal ganglia without associated metabolic derangement in a patient with OTC deficiency has not been described previously.

Metabolic stroke has been described in patients with urea cycle defects, including several patients with OTC deficiency and in one patient with carbamyl phosphate synthetase (CPS) deficiency (MIM 237300) [4, 5, 13, 15, 20]. Each of these patients presented with hemiparesis and hyperammonemia in the range of $300-400 \mu \mathrm{M}$, and all had a CT or MRI pattern consistent with infarction

Fig. 2A-D Results of neuroimaging studies. A Axial fluid attenuated inversion recovery (FLAIR) MR scan on hospital day 2 demonstrates mild increased signal from the putamina (long arrows) and more subtle increased signal from the head of the caudate nuclei (short arrows) bilaterally. The globi pallidi (arrow heads) display normal signal. B Non-contrast axial CT scan on hospital day 4 demonstrates subtle decreased attenuation from region of the putamina (long arrows). C Axial fluid attenuated inversion recovery (FLAIR) MR scan on hospital day 9 demonstrates progressive increase in signal from the putamina (long arrows) and head of the caudate nuclei (short arrows) over 7 days. D Contrast-enhanced T1-weighted MR scan shows mild local mass effect associated with the putaminal abnormalities, no enhancement is seen (long arrows). The globi pallidi (arrow heads) are normal on both FLAIR and T1-weighted images 
Table 2 CNS regions affected in metabolic stroke

\begin{tabular}{|c|c|c|c|c|c|}
\hline Disorder & Cortex & Cerebellum & $\begin{array}{l}\text { Basal ganglia/ } \\
\text { globus pallidus }\end{array}$ & $\begin{array}{l}\text { Basal ganglia/caudate } \\
\text { and putamen }\end{array}$ & Refs. \\
\hline Current patient & - & - & - & + & \\
\hline OTC & + & - & - & - & {$[4,5,13,15]$} \\
\hline CPS & + & - & - & - & [20] \\
\hline Propionicacidemia & - & + & + & - & {$[8,9,10]$} \\
\hline Methylmalonicacidemia & - & + & + & - & {$[3,11,12,22]$} \\
\hline Glutaricacidemia type I & - & - & - & + & {$[7,14]$} \\
\hline Isovalericacidemia & - & + & - & - & [6] \\
\hline 3-methylcrotonyl CoA carboxylase & + & - & - & - & [21] \\
\hline Leighsyndrome & + & + & + & + & {$[16,19]$} \\
\hline MELAS & + & + & + & + & [19] \\
\hline
\end{tabular}

OTC ornithine transcarbamylase deficiency; CPS carbamyl phosphate synthetase deficiency; $M E L A S$ mitochondrial myopathy encephalopathy lactic acidosis stroke-like episodes

or ischemia of the cerebral cortex. None of these patients had evidence of damage to the basal ganglia as seen in our patient. Interestingly, the four patients with OTC deficiency were heterozygous females, and the diagnosis of OTC deficiency was made after the onset of strokelike symptoms $[4,5,13,15]$.

Stroke-like symptoms are relatively uncommon in patients with inborn errors of metabolism. Excluding mitochondrial disorders, such as Leigh syndrome (MIM 256000) and MELAS (MIM 540000), metabolic stroke has been reported in approximately 20 patients. There appears to be some specificity for certain affected regions of the brain, depending on the type of metabolic disorder (Table 2). Patients with organic acidemias, including methylmalonic acidemia (MIM 251000) and propionic acidemia (MIM 606054), have been described with acute injuries to the basal ganglia; however, of note, these patients had injuries to the globus pallidus, not the neostriatum $[8,11,12,22]$. In addition, all but one of these patients had metabolic decompensation with significant acidosis at the time of presentation [8]. The recent observation of a patient with methylmalonic acidemia who suffered a metabolic stroke while in good metabolic control almost 5 years after liver transplantation suggests that factors other than hepatic correction of enzyme activity may predispose to metabolic stroke [3].

A number of mechanisms have been proposed to account for the neurological abnormalities associated with metabolic stroke. These include the accumulation of toxic substances within the basal ganglia [22], hypoxic-ischemic (watershed) injury [13, 15], severe ketoacidosis [11], and accumulation of glutamine in the brain [20]. The basal ganglia are thought to be especially susceptible to hypoxic and/or ischemic damage, and necrosis of the globus pallidus is a frequent acute complication of exposure to both carbon monoxide and cyanide [11], both of which were ruled out in our patient. Given this increased requirement for oxidative metabolism, it has been hypothesized that basal ganglia injuries in patients with metabolic stroke are due to a deficiency of mitochondrial energy metabolism [9]; however, the exact mechanism of injury to the brain is unknown.
In summary, metabolic stroke is a recognized complication of several different inborn errors of metabolism. The etiology of this neurological insult is unknown, but possibly involves accumulation of toxic intermediates or disruption of mitochondrial function. To our knowledge, this is the first report of metabolic stroke in OTC deficiency involving the basal ganglia and occurring in the absence of acute metabolic decompensation. Our report suggests that patients with inborn errors presenting with neurological symptoms and normal biochemical parameters should be evaluated carefully for the development of metabolic stroke. Similarly, patients with acute, unexplained neurological insults should be evaluated for inborn errors of metabolism. This case adds to the expanding list of disorders with a variety of presentations that can be classified as metabolic stroke.

\section{References}

1. Anadiotis G, Ierardi-Curto L, Kaplan PB, Berry GT (2001) Ornithine transcarbamylase deficiency and pancreatitis. J Pediatr 138: 123-124

2. Brusilow SW, Maestri NE (1996) Urea cycle disorders: diagnosis, pathophysiology, and therapy. Adv Pediatr 43: 127-170

3. Chakrapani A, Sivakumar P, McKiernan PJ, Leonard JV (2002) Metabolic stroke in methylmalonic acidemia five years after liver transplantation. J Pediatr 140: 261-263

4. Christodoulou J, Qureshi IA, McInnes RR, Clarke JT (1993) Ornithine transcarbamylase deficiency presenting with strokelike episodes. J Pediatr 122: 423-425

5. De Grauw TJ, Smit LM, Brockstedt M, Meijer Y, vd Klei-von Moorsel J, Jakobs C (1990) Acute hemiparesis as the presenting sign in a heterozygote for ornithine transcarbamylase deficiency. Neuropediatrics 21: 133-135

6. Fischer AQ, Challa VR, Burton BK, McLean WT (1981) Cerebellar hemorrhage complicating isovaleric acidemia: a case report. Neurology 31: 746-748

7. Goodman SI, Norenberg MD, Shikes RH, Breslich DJ, Moe PG (1977). Glutaric aciduria: biochemical and morphologic considerations. J Pediatr 90: 746-750

8. Haas RH, Marsden DL, Capistrano-Estrada S, Hamilton R, Grafe MR, Wong W, Nyhan WL (1995) Acute basal ganglia infarction in propionic acidemia. J Child Neurol 10: 18-22

9. Hamilton RL, Haas RH, Nyhan WL, Powell HC, Grafe MR (1995) Neuropathology of propionic acidemia: a report of 
two patients with basal ganglia lesions. J Child Neurol 10: 2530

10. Harding BN, Leonard JV, Erdohazi M (1991) Propionic acidaemia: a neuropathological study of two patients presenting in infancy. Neuropathol Appl Neurobiol 17: 133-138

11. Heidenreich R, Natowicz M, Hainline BE, Berman P, Kelley RI, Hillman RE, Berry GT (1988) Acute extrapyramidal syndrome in methylmalonic acidemia: "metabolic stroke" involving the globus pallidus. J Pediatr 113: 1022-1027

12. Korf B, Wallman JK, Levy HL (1986) Bilateral lucency of the globus pallidus complicating methylmalonic acidemia. Ann Neurol 20: 364-366

13. Lacey DJ, Duffner PK, Cohen ME, Mosovich L (1986) Unusual biochemical and clinical features in a girl with ornithine transcarbamylase deficiency. Pediatr Neurol 2: 51-53

14. Leibel RL, Shih VE, Goodman SI, Bauman ML, McCabe ER, Zwerdling RG, Bergman I, Costello C (1980) Glutaric acidemia: a metabolic disorder causing progressive choreoathetosis. Neurology 30: 1163-1168

15. Mamourian AC, du Plessis A (1991) Urea cycle defect: a case with MR and CT findings resembling infarct. Pediatr Radiol 21: $594-595$

16. Medina L, Chi TL, DeVivo DC, Hilal SK (1990) MR findings in patients with subacute necrotizing encephalomyelopathy (Leigh syndrome): correlation with biochemical defect. AJR Am J Roentgenol 154: 1269-1274
17. Msall M, Batshaw ML, Suss R, Brusilow SW, Mellits ED (1984) Neurologic outcome in children with inborn errors of urea synthesis. Outcome of urea-cycle enzymopathies. N Engl J Med 310: 1500-1505

18. Picca S, Dionisi-Vici C, Abeni D, Pastore A, Rizzo C, Orzalesi M, Sabetta G, Rizzoni G, Bartuli A (2001) Extracorporeal dialysis in neonatal hyperammonemia: modalities and prognostic indicators. Pediatr Nephrol 16: 862-867

19. Shoffner JM (2001) Oxidative phosphorylation diseases. In: Scriver CM, Beaudet AL, Sly WS, Valle D, Childs B, Kinzler KW, Vogelstein B (eds) The metabolic and molecular bases of inherited disease. McGraw-Hill, New York, pp 2367-2423

20. Sperl W, Felber S, Skladal D, Wermuth B (1997) Metabolic stroke in carbamyl phosphate synthetase deficiency. Neuropediatrics 28: 229-234

21. Steen C, Baumgartner ER, Duran M, Lehnert W, Suormala T, Fingerhut SR, Stehn M, Kohlschutter A (1999). Metabolic stroke in isolated 3-methylcrotonyl-CoA carboxylase deficiency. Eur J Pediatr 158: 730-733

22. Thompson GN, Christodoulou J, Danks DM (1989) Metabolic stroke in methylmalonic acidemia. J Pediatr 115: 499-500

23. Uchino T, Endo F, Matsuda I (1998). Neurodevelopmental outcome of long-term therapy of urea cycle disorders in Japan. J Inherit Metab Dis 21(Suppl 1): 151-159 